\title{
Las redes sociales como herramienta para la divul- gación de fondos documen- tales de bibliotecas. Estudio de caso de la cuenta en Twitter de la JFK Library
}

\author{
Pere Franch \\ Elena Yeste*
}

Artículo recibido: 23 de diciembre de 2014 Artículo aceptado: 9 de septiembre de 2016

\section{Resumen}

Se expone el uso que la JFK Library hace de las redes sociales para dar a conocer su actividad, se presenta la cuenta de Twitter@JFKsaid, creada en 2010 para conmemorar los 50 años de la llegada de J. F. Kennedy al poder y se estudia el uso de dicha cuenta para difundir los fondos documentales digitalizados del centro. El resultado pone en evidencia que la institución aprovecha poco el potencial de dicha cuenta para divulgar sus documentos digitalizados, entre los que se observa una ínfima presencia de videos frente a una mayor cantidad de fotos y archivos de audio. Como conclusiones principales se constata que Twitter ofrece enormes posibilidades para potenciar la difusión del conocimiento y

Universidad Ramon Llull, España. perefp@blanquerna.url.edu elenayp@blanquerna.url.edu

INVESTIGACIÓN BIBLIOTECOLC 
el intercambio de información y documentación, que la cuenta@JFKsaid tiene más valor rememorativo que divulgativo y que su función es sobre todo autoreferencial.

Palabras clave: Redes sociales; Twitter; Comunicación; Bibliotecas; Bibliotecas especializadas; Archivos digitales; Servicios bibliotecarios; Difusión de fondos; Efemérides; Kennedy.

\section{Abstract}

Social media as a tool to disseminate library contents. Case study of the JFK Library Twitter account Pere Franch and Elena Yeste

After describing the use of social media services (SMS) by JFK Library, we specifically study the use of the Twitter account @JFKsaid, created initially to commemorate the 50th anniversary of John F. Kennedy's election as President of the United States of America. Our central research question asks how the JFK Library uses this Twitter account to disseminate digitalized documents (video, audio, text and photo) stored in its archives. Our findings show that Twitter is rarely used as a link to the JFK Library website and also that the number of video documents broadcast is insignificant when compared to the dissemination of audio files or photos. Even though, the use of Twitter by the JFK Library is focused largely on the self-referential task of commemorating President Kennedy and his legacy, our study confirms Twitter's considerable potential as a medium for disseminating knowledge and sharing information and documentation.

Keywords: Social media; Twitter; Communication; Libraries; Specialized Libraries; Digital Archives; Library Services; Dissemination of Stored Documents; Anniversaries; Kennedy.

Este trabajo forma parte del proyecto "Información política, Twitter y democracia. El periodismo en el entorno de los medios sociales" (Proyecto de I+D+i. Ministerio de Economía y Competitividad del Gobierno de España) CSO2014-52283. 


\section{INTRODUCCIÓN}

T a National Archives and Records Administration (NARA) de los Estados LUnidos es la agencia federal encargada de conservar y poner a disposición de la población norteamericana el material documental generado por el gobierno federal a lo largo de la historia. Al frente de ella está el Archivero de los Estados Unidos (cargo que ejerce David Ferriero desde noviembre de 2009, http://blogs.archives.gov/aotus/), nombrado por el presidente y ratificado por el Senado. En su página web (http://www.archives.gov), la NARA informa que sólo se archiva entre el 1 y el 3 por ciento del material que genera el gobierno. A pesar de ello, las cifras son abrumadoras: unos 3000 empleados a tiempo completo o parcial trabajan en las 36 instalaciones que la NARA tiene distribuidas en todo el país para gestionar, según sus propios datos, 10 billones de páginas de textos, 12 millones de mapas y planos, 25 millones de fotografías y gráficos, 24 millones de fotografías aéreas, 300000 rollos de película, 400000 documentos de video y de audio y 133 terabytes de información digitalizada.

De la NARA también dependen las 13 bibliotecas-museo presidenciales de los Estados Unidos, una red de centros de documentación dedicados a conservar y difundir el legado de los presidentes desde Herbert Hoover (1929-1933) hasta la actualidad. Según la NARA, su función es "preservar y proporcionar acceso a material histórico, apoyar la investigación y la creación de programas y exposiciones interactivas que educan e inspiran" (http:// www.archives.gov/presidential-libraries/ Traducción propia). Una de estas 13 bibliotecas es la dedicada a John Fitzgerald Kennedy, con sede en Boston, que declara en su página web (http://www.jfklibrary.org) que tiene por objetivo "avanzar en el estudio y comprensión de la vida y la carrera del presidente Kennedy y los tiempos en que vivió”. Existen más bibliotecas dedicadas a otros presidentes, pero no dependen de la NARA sino de fundaciones privadas, con participación, en algunos casos, de gobiernos estatales.

\section{La JFK Library y las redes sociales}

La JFK Library and Museum (a partir de aquí, JFK Library) se fundó el 5 de diciembre de 1963 , trece días después del asesinato del $35^{\circ}$ presidente de los Estados Unidos. Al principio una fundación privada sin ánimo de lucro, se incorporó plenamente al sistema de bibliotecas presidenciales de la NARA el 20 de octubre de 1979.

En pleno siglo XXI, con la aparición de las tecnologías 2.0, los responsables de la JFK Library han recurrido a las redes sociales para difundir su 
material documental y dar a conocer el legado de Kennedy. La JFK tiene abiertos canales en Facebook, Instagram, Pinterest, Tumblr, Youtube, iTunes y Twitter. En Facebook (https://www.facebook.com/JFKLibrary) tiene cuenta activa desde el 29 de mayo de 2008 y en noviembre de 2014 tenía más de 56000 seguidores. En Instagram (http://instagram.com/jfklibrary) la primera foto se subió el 9 de julio de 2013 y en noviembre de 2014 exponía 132 fotos y tenía 1023 seguidores. Para la misma fecha se ofrecían en Pinterest (http://www.pinterest.com/jfklibrary/) 124 imágenes distribuidas en 10 tableros. La cuenta en Tumblr (http://jfklibrary.tumblr.com) se abrió en febrero de 2012 y en octubre de 2014 había publicado un total de 411 posts. El canal de Youtube (https://www.youtube.com/user/JFKLF) se creó el 2 de mayo de 2007 y al 20 de noviembre de 2014 contabilizaba más de 7700 suscriptores y más de tres millones de visualizaciones de los 187 videos que contiene, distribuidos en ocho listas de reproducción. En iTunes el centro dispone de dos canales de podcasts, uno con 50 archivos de audio de actividades organizadas por la institución (https://itunes.apple.com/us/podcast/ jfk-library-audio-podcast/id524823939?mt=2) y otro con 18 archivos de audio con sendos discursos o declaraciones de Kennedy (https://itunes.apple. com/us/podcast/jfk-library-museum-john-f./id698789762?mt=2).

\section{La JFK y Twitter}

La JFK Library tiene abiertas tres cuentas en Twitter: la institucional @JFKLibrary (https://twitter.com/JFKLibrary) es la cuenta institucional; se abrió en abril de 2009 y ofrece información sobre todas las actividades de la entidad. Las otras dos son cuentas personales creadas específicamente para rememorar la actividad del presidente Kennedy y de su esposa. La de esta última, @JBK1960 (https://twitter.com/JBK1960), sólo estuvo activa del 15 de septiembre al 7 de noviembre de 2010, periodo en el que publicó 98 tuits para rememorar la actividad periodística de Jacqueline Bouvier Kennedy cincuenta años antes: presentaba extractos de la columna titulada "Campaign wife” que publicó en la prensa escrita durante la campaña presidencial de 1960.

La cuenta dedicada al presidente, @JFKsaid, publicó el primer tuit el 2 de enero de 2010, al cumplirse exactamente 50 años del anuncio oficial de que iba a presentarse a las elecciones presidenciales. Una semana antes, la JFK Library anunciaba así aquella iniciativa: "a partir de la selección de calendarios de campaña, comunicados de prensa, anotaciones en diarios personales y periódicos de los archivos de la biblioteca Kennedy, la página de Twitter Kennedy1960 permite a los usuarios de Internet en todo el mundo seguir el 
día a día del calendario de campaña de John F. Kennedy en su camino hacia la Casa Blanca" (JFK Library and Museum, 2009). Aquella cuenta era @ Kennedy1960, aunque la dirección actual es @JFKsaid (https://twitter.com/ JFKsaid). En noviembre de 2014 tenía más de 36400 seguidores y había publicado más de 4400 tuits.

El origen de esta cuenta fue la conmemoración del 50 aniversario de su campaña, elección, llegada al poder y posterior asesinato. Por ello, después del 22 de noviembre de 2013 permaneció inactiva más de medio año, hasta que el 10 de junio de 2014 reanudó la publicación regular de tuits que reproducen frases de Kennedy e insertan imágenes o videos de hechos acaecidos en su época, siempre coincidiendo con su efeméride. Megan Piccirillo, encargada de la gestión de las redes sociales (social media manager) de la JFK Library, remarca en una entrevista realizada para este estudio el carácter rememorativo de la cuenta y su voluntad de mantener el legado del presidente:

Después del aniversario de 22 de noviembre, nos tomamos unos meses para volver a evaluar nuestra estrategia de medios sociales. Una de las cuestiones que notamos fue que las citas y discursos del presidente Kennedy eran los tuits que más compartían nuestros seguidores de @JFK_1963. Rachel Flor, nuestra responsable de Comunicaciones y Medios Digitales, tuvo la idea de usar esas citas de una manera diferente formulando la pregunta: “¿Cómo reaccionaría JFK a los problemas de hoy?”, y convertirla en una nueva cuenta de Twitter: @JFKsaid. En lugar de perder todos nuestros seguidores de la @JFK_1963, simplemente cambiamos el nombre de usuario por @JFKsaid. Estábamos bastante seguros de que el público de@JFK_1963 estaría interesado en nuestro nuevo proyecto. Elegimos el 10 de junio de 2014 para poner en marcha la nueva cuenta de Twitter porque era el aniversario de uno de los discursos más famosos del presidente Kennedy, su discurso sobre la paz en la American University.

La creación por parte de la JFK Library de un servicio de comunicación y difusión a través de Twitter del legado del presidente Kennedy se lleva a cabo, por tanto, a partir de la utilización de la figura concreta de la efeméride. La efeméride trata un tipo de producto informativo-documental (es decir, que conjuga información y documentación) que cuenta con amplia tradición y uso extendido en el contexto de los bibliotecarios y documentalistas que trabajan en medios de comunicación (Giménez Rayo y Guallar, 2014; Guallar, 2011) pero que ha sido menos utilizado por estos mismos profesionales en bibliotecas, de ahí que el caso que aquí nos ocupa sea una iniciativa que puede ser novedosa en este sector. 


\section{OBjeto DE LA INVESTIGACión y METODOLOGÍA}

El presente artículo pretende analizar el uso que la JFK Library hizo de la cuenta @JFKsaid para difundir su fondo documental a través de la red social Twitter. Se ha acotado la observación a los tuits publicados durante el periodo que coincide exactamente con el cincuenta aniversario de la presidencia de Kennedy: desde el 20 de enero de 2010, efeméride de su toma de posesión, hasta el 22 de noviembre de 2013, aniversario de su asesinato. En total se han analizado 3253 tuits, en los que se ha estudiado en cuántos se facilitaba acceso a los fondos documentales de la institución y qué tipo de documentos digitalizados se ofrecía a los usuarios: textos, fotos, videos o archivos sonoros. Se ha observado también si este acceso a los fondos se facilitaba mediante un enlace a la página web de la biblioteca o bien se incrustaba el documento en el texto de los tuits.

La finalidad es observar el uso de las redes sociales, en concreto de la red de microblogging Twitter, para la difusión de los fondos documentales de las bibliotecas especializadas. Comprobar qué uso hacen de Twitter los responsables de la JFK Library puede proporcionar elementos para calibrar la oportunidad de esta iniciativa y plantear la posibilidad de aplicarla en otros contextos.

El análisis se ha llevado a cabo aplicando una metodología de investigación descriptiva cuantitativa y la técnica del análisis de contenido de los tuits de la cuenta a estudiar. Para ello se ha extraído de la cuenta el contenido textual de los 3253 tuits que se querían analizar y se han observado aquellos que incorporaban en el texto un enlace (encabezado por el indicador $b t t p$ ) y a la vez aquellos que directamente adjuntaban a los 140 caracteres una fotografía o un archivo de video. Una vez identificados los 530 tuits con enlace, se ha observado a dónde llevaban los 577 enlaces publicados (hay tuits con dos, tres y hasta cuatro enlaces en el mismo texto) y se han clasificado según llevaran a archivos de texto, de video, de audio o fotografías. Una vez hecha esta clasificación, se ha procedido a describir las características de los documentos enlazados. De manera complementaria, se ha mantenido una entrevista con la responsable de la cuenta.

El uso de una metodología basada en la descripción cuantitativa y el análisis de contenido se justifica por el hecho de que precisamente aquello que se pretende con esta investigación es conocer un aspecto del contenido de los tuits (si tienen o no un enlace web) y, una vez identificados estos mensajes de microblogging, observar su uso para difundir el fondo documental de la JFK Library. Específicamente se buscan datos cuantitativos: números de tuits con enlaces y qué tipo de archivo proporcionan al usuario de la red. No se ha 
observado qué uso hacen los usuarios, sino que nos hemos centrado en el uso que de esta red social hacen los gestores de la biblioteca, por ello nos parece también pertinente una entrevista con su responsable. La mayor parte de la literatura sobre Twitter citada en este artículo incluye también una observación del contenido de los mensajes y una clasificación cuantitativa de dicho contenido, siempre en función de aquello que se pretende estudiar.

\section{El uso de TwitTer COMO heRramienta PARA LA DivUlgación DEL CONOCIMIENTO EN ARCHIVOS Y BIBLIOTECAS}

Los archivos y las bibliotecas tienen entre sus principales cometidos la preservación de la memoria colectiva. La combinación de Internet y los nuevos medios interactivos y multimedia ofrece a estas instituciones nuevas oportunidades para la construcción de la memoria:

Mientras que los archivos de los medios de comunicación de la época de la difusión se almacenaban en el espacio físico del archivo o la biblioteca, sujetos a las condiciones materiales de catalogación, clasificación y recuperación (es decir, acceso), la conectividad es de una importancia primordial para el archivo digital como un medio inequívocamente de masas. (Hoskins, 2009: 6)

De esta manera, los archivos multimedia contribuyen a la divulgación del conocimiento haciéndolo accesible y compartible: "el archivo multimedia digital es un archivo en red online -un interarchivo- que es, por defecto, accesible y compartible. Como señala Wolfgang Ernst, lo que define el archivo multimedia es su hipervinculabilidad e interconectividad". (Pinchevski, 2011: 255)

El uso de la tecnología 2.0 en general en el ámbito de los servicios que ofrecen las bibliotecas ha sido ampliamente estudiado desde hace ya unos años (Stephens, 2006; Casey y Savstinuk, 2007; Kroski, 2008; Aharony, 2009; Chua y Goh, 2010). Algunos trabajos más recientes abordan el tema centrándose en las bibliotecas universitarias (Tripathi y Kumar, 2010; Mahmood y Richardson, 2011; Boateng y Liu, 2013; Alonso Arévalo et al., 2014).

En cuanto al uso de, específicamente, las redes sociales en bibliotecas sólo digitales, es reseñable el trabajo de Xie y Stevenson (2014a), quienes, tras analizar diez bibliotecas, constatan tres problemas: la falta de normas o criterios generales, la escasa interacción con los usuarios y la ausencia de objetivos educativos. También ponen en evidencia que Twitter, junto a Facebook, es la red social más utilizada por las bibliotecas digitales analizadas. Esta es 
también una de las conclusiones de Boateng y Liu (2013: 130), quienes han realizado un estudio cuantitativo del uso de las aplicaciones web 2.0 en 100 bibliotecas universitarias norteamericanas: todas ellas usan Facebook y Twitter, por lo que los llamados Social Network Services (SNS) son la herramienta más ampliamente utilizada.

Otros estudios (Xie y Stevenson, 2014b; Del Bosque, Leif y Skarl, 2012; Aharony, 2010) realizan una aproximación a las funciones de Twitter como herramienta de microblogging en bibliotecas. Xie y Stevenson (2014b: 2) establecen la siguiente categorización: Information-Digital Library (DL)-problem Tweets, que informan acerca de problemas relacionados con la accesibilidad o el mantenimiento del sitio web; Promotion-DL-collection Tweets, que anuncian la publicación de un elemento recién agregado a una colección digital o de una nueva colección; Social connection-DL-interaction Tweets, fruto de la interacción con los usuarios de la cuenta; Related resources-DL Tweets, con los que la biblioteca se relaciona y conecta con otras bibliotecas y fondos digitalizados para construir una comunidad en línea; Social identity-DL-collection Tweets, con los que la biblioteca configura su propia identidad social a través de la singularidad de su colección digital. Estas funciones entroncan con las propias y generales de las redes sociales: "El suministro de información, marketing / promoción, conexiones entre usuarios, y el intercambio de información, todas ellas son diferentes funciones de los medios sociales relacionadas con las bibliotecas digitales" (Xie y Stevenson, 2014a: 516).

La JFK Library, como el resto de bibliotecas presidenciales, está dedicada a una figura política; por lo tanto, el grueso de la información que difunde es de contenido político. El estudio de la utilización de la red social Twitter en el ámbito de la comunicación política se ha centrado en ver qué uso hacían de ella los usuarios, como se constata en los trabajos de Tumasjan et al. (2010) o Small (2011), o bien en el uso que los dirigentes políticos hacen de ella para difundir su mensaje: Ammann (2010), Golbeck, Grimes y Rogers (2010), Glassman, Straus y Shogan (2010), Aharony (2012), Adams y McCorkindale (2013) y Franch (2013). Estos autores estudian el contenido de los mensajes que los dirigentes políticos envían a los usuarios de Twitter. Las principales conclusiones a las que llegan son que los líderes usan esta red social para sortear los medios de comunicación y comunicarse directamente con su público con el objetivo de dar a conocer su actividad y también, sobre todo, para aumentar y canalizar el tráfico de los usuarios hacia otras páginas web; también concluyen que establecen muy poca interacción con los usuarios. 


\section{Resultados}

De los 3253 tuits analizados, sólo 530 incorporan algún enlace entre los 140 caracteres publicados. Esta cifra supone el 16.3 \% del total. La mayoría contiene un solo enlace (489), hay 36 con dos, cuatro con tres enlaces y uno solo con cuatro (Tabla 1 y Figura 1). En total, se han observado 577 enlaces publicados en la cuenta en el periodo analizado. Casi todos ellos (97 \%) enlazan con documentación perteneciente al fondo de la JFK Library; el resto, 17 enlaces ( $3 \%)$, lleva a páginas externas que en algunos casos también son obra de la misma JFK, pero no conectan con materiales de su fondo documental.

\begin{tabular}{|c|c|c|c|c|c|c|c|c|c|c|}
\hline & & & & & & & & & & TOTAL \\
\hline $\begin{array}{l}\text { Tuits } \\
\text { analizados }\end{array}$ & & & & & & & & & & 3.253 \\
\hline Tuits con & con & enlace & $\begin{array}{l}\text { con } \\
\text { enl: }\end{array}$ & & $\begin{array}{l}\text { cor } \\
\mathrm{cnl}\end{array}$ & & & & & \\
\hline enlace & 489 & $92.3 \%$ & 36 & $6.8 \%$ & 4 & $0.75 \%$ & 1 & $0.18 \%$ & 530 & $16.3 \%$ \\
\hline Enlaces & 489 & & 72 & & 12 & & 4 & & & 577 \\
\hline
\end{tabular}

Tabla 1. Tuits con enlaces de la cuenta @JFKsaid. Se han analizado en total 3253 tuits de la cuenta @JFKsaid, publicados entre el 20 de enero de 2011 y el 22 de noviembre del 2013. De ellos, 530 (el 16.3\%) tienen algún enlace: la mayor parte, 489 (el 92.3\%) tienen un enlace; 32 (el 6.8\%) tienen dos; cuatro tienen tres (el 0.75\%) y sólo uno (el $0.18 \%$ ) tiene cuatro, lo que nos lleva a un total de 577 enlaces, a documentos del fondo de la JFK Library and Museum y, en muy pocos casos, a páginas web externas.

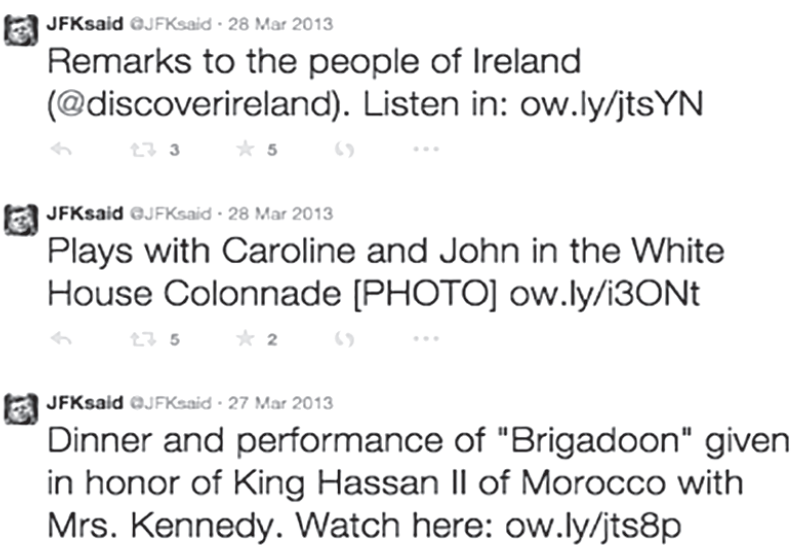


A través del enlace el usuario puede acceder a cuatro tipos de documento: fotos, archivos de audio, documentos de texto o videos (Tabla 2), cuya ficha responde en todos los casos al mismo formato. A la izquierda aparece el documento en sí (foto o documento de texto digitalizado y reproductor de video o de audio, Figura 2) y a la derecha, distribuidos verticalmente, los siguientes campos con información sobre el documento: número de registro, título, fecha en la que fue creado el material, autor, descripción del contenido, descripción física del documento original, tipo de documento, temas, personajes, organismos o entidades, lugares, título de la carpeta, título y número de la serie, colección, propietario de los derechos y si está sujeto a copyright o no, créditos y un número de identificación digital. En todos los documentos aparecen algunos de los campos mencionados, con las especificidades según el tipo.

\begin{tabular}{|r|r|r|}
\hline Enlaces & $\mathbf{5 7 7}$ & \\
\hline Foto & 263 & $45.6 \%$ \\
\hline Audio & 177 & $30.7 \%$ \\
\hline Texto & 100 & $17.3 \%$ \\
\hline Video & 20 & $3.4 \%$ \\
\hline Externos & 17 & $3 \%$ \\
\hline
\end{tabular}

Tabla 2. Número de documentos enlazados en la cuenta @JJFksaid. De todos los enlaces publicados por @.JFKsaid durante el periodo estudiado, sólo 17 dirigen a páginas web externas; el resto, 97 \%, dirigen a fotos, archivos de audio, textos escaneados o videos del fondo documental de la JFK Library and Museum.

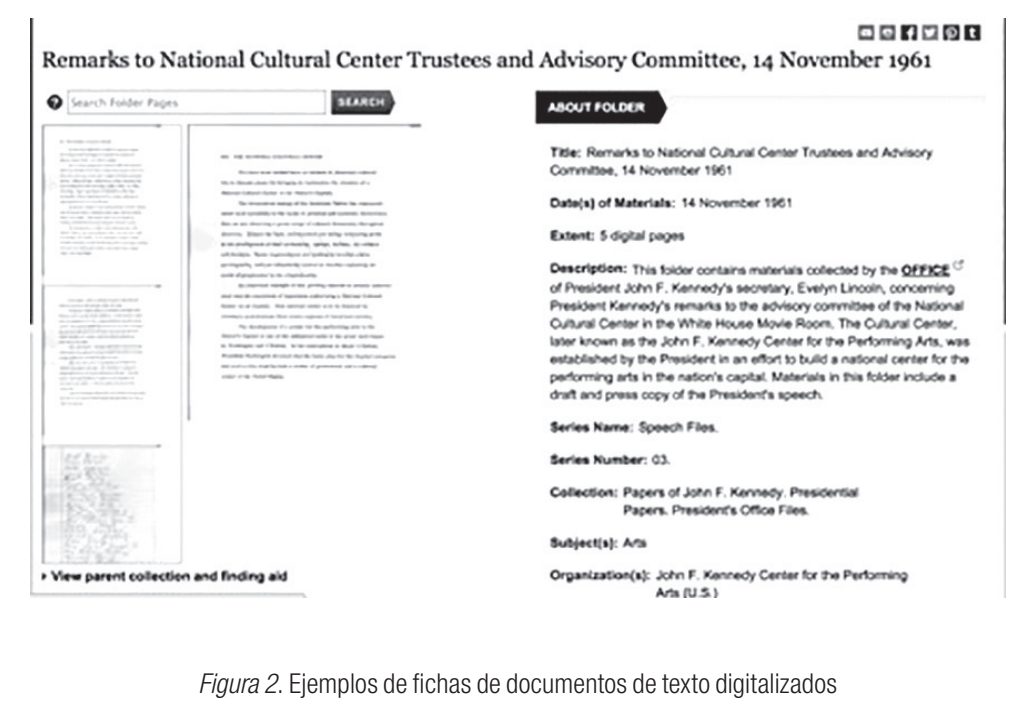




\section{Fotografia}

En el caso de las fotografías, el nombre del fotógrafo constituye en muchos casos un enlace a la lista de la JFK Library con todas las fotografías del mismo autor. Los temas, los personajes, las organizaciones y las colecciones suelen presentarse también como enlace al resto de documentos clasificados bajo los mismos nombres o etiquetas. Es interesante notar que también se ofrece la herramienta de aumento de la imagen mediante el deslizamiento del cursor por encima de la fotografía. Las páginas pueden ofrecer, del mismo evento, desde una sola fotografía hasta carpetas con decenas de ellas, presentadas en un visor scroll que permite elegir cualquiera, ampliarla y avanzar y retroceder en la carpeta.

\section{Documentos de audio}

La mayoría son declaraciones del presidente, fragmentos de discursos, intervenciones o conferencias. La tipología es la misma que para el resto de documentos, con algunas peculiaridades: en la página aparece el reproductor de audio con la duración del documento. En la mayoría de los casos se ofrece al usuario la posibilidad de descargar el archivo, de forma automática y gratuita siempre que no esté sujeto a derechos de autor.

\section{Documentos de texto}

Suelen ser transcripciones, cartas recibidas o enviadas, textos manuscritos, notas o incluso reproducciones de otro tipo de documentos, siempre escaneados y con la misma información acerca de ellos que la referida anteriormente, con variaciones en su extensión. Se da a conocer el número de páginas cuando el documento lo justifica, y siempre se suele presentar con un visor scroll que permite elegir la página que se quiere visualizar, ampliarla y avanzar o retroceder. Cabe decir que en algunos casos el enlace lleva directamente a un documento en formato JPG e incluso a algunos en formato PDF.

\section{Videos}

La presencia de videos es muy escasa. La página de videos de la JFK mantiene la misma tipología, con estas peculiaridades: a la izquierda aparece el reproductor del video y a la derecha, además de los campos ya mencionados, figuran la duración y el metraje de la grabación (Figura 3). 

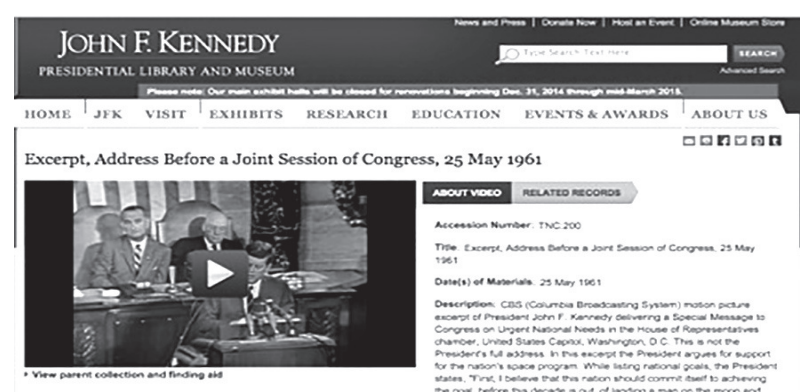

nestrveso neurzo naconos

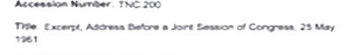

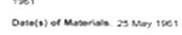

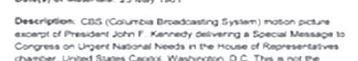

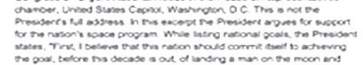

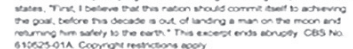

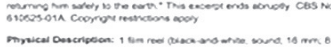

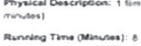

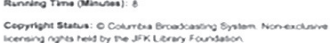

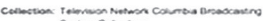

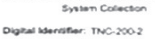

wetecents seose

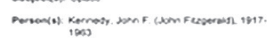

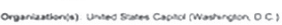

Figura 3. Ejemplo de ficha de documento de video

\section{Enlaces externos}

Sólo 17 de los 577 enlaces no llevan a un documento digitalizado del fondo de la JFK Library, pero muchos de ellos llevan a otra página creada por la misma biblioteca. Así, en siete casos se enlaza con microsites creadas y hospedadas en la página web jfklibrary.org. En tres casos se enlaza con páginas de la American Presidency Project (donde se archivan discursos de los presidentes norteamericanos en su sección Public Papers of the Presidents). Se dan, además, los siguientes casos particulares: el último tuit enlaza con una página de la biblioteca creada especialmente para conmemorar el 50 aniversario de su asesinato, titulada An idea lives on. Hay un enlace a la página de Facebook de la JFK, uno a la cuenta de Tumblr de los National Archives, uno a un video de Youtube, uno que lleva a la extensión de Twitter Twitlonger y uno a la revista Sports Illustrated.

Se ha observado que en 55 casos los enlaces llevan directamente a la cuenta de servicios y analítica HootSuite, de acortamiento de enlaces (http://ow.ly/user/JFKsaid), que da acceso a 11 fotos y a 44 textos digitalizados en lugar de dirigir a las fichas del fondo documental de la biblioteca.

Cabe señalar, además, que en sólo 10 casos de los estudiados se incorpora un archivo JPG como objeto adherido al tuit sin enlace; en ellos se reproducen tanto imágenes como documentos de texto. 


\section{ConClusiones}

El estudio de la cuenta @JFKsaid permite constatar las posibilidades que ofrece Twitter para potenciar la difusión del conocimiento, el intercambio y la discusión de la información y documentación en posesión de bibliotecas y archivos con fondos digitalizados, y llegar a un amplio número de usuarios conectados con los que, además, es posible interactuar.

La JFK Library utiliza la figura de la efeméride en la cuenta @JFKsaid más para reforzar la figura del presidente y consolidar su presencia en el imaginario colectivo de la sociedad norteamericana que para difundir sus fondos documentales. Sólo el 16.3 \% de los tuits analizados facilitan el acceso a material documental de la biblioteca; el resto rememoran algún aspecto de la vida, el legado y la actividad política del presidente. Megan Piccirillo reafirma la voluntad de la institución de difundir las ideas y la obra de Kennedy entre el público actual:

La cuenta de Twitter @JFKsaid conecta ideas y discursos del presidente Kennedy con lo que está pasando en el mundo de hoy. Es una gran manera de llevar su legado a las audiencias más jóvenes en las redes sociales y demostrar lo poderosas que sus palabras siguen siendo cincuenta años más tarde. Tratamos de ser lo más equilibrados que podemos, y sólo recogemos los temas y noticias que tengan sentido para nuestra institución.

Es significativa la ínfima cantidad de archivos de video del fondo bibliotecario que se ofrecen a través de la cuenta en contraste con la gran cantidad de documentos de audio y fotografías. Una explicación plausible es que en el periodo 1960-1963 la tecnología audiovisual estaba en su desarrollo inicial, mientras que el registro de archivos sonoros y fotográficos estaba mucho más extendido. En la web de la JFK Library se puede consultar el número total de archivos digitalizados según el formato: hay 124 videos frente a 1461 archivos sonoros, 14735 fotografías y 9861 carpetas con documentos escaneados que contienen de una a decenas de páginas digitalizadas. Por tanto, la variedad de archivos digitales a los que se tiene acceso a través de la cuenta @JFKsaid guarda cierta relación proporcional con la variedad de formatos de archivos del fondo, consecuencia a su vez de la realidad de la comunicación política en la época de Kennedy: los medios preponderantes eran la prensa y la radio, mientras que la televisión era todavía incipiente en el ámbito político. Cabe recordar que el debate Nixon-Kennedy del 26 de septiembre de 1960 fue el primero televisado y que la costumbre de retransmitir en directo las ruedas de prensa presidenciales llegó precisamente con Kennedy: su primera conferencia de prensa como 
inquilino de la Casa Blanca fue retransmitida en directo por televisión, el 25 de enero de 1961. Sin embargo, 50 años después la cuenta de Twitter, paradójicamente, da acceso a la transcripción de aquella rueda de prensa pero no al video, a pesar de que este existe en los fondos catalogados de la biblioteca.

La poca presencia de enlaces a otras páginas web que no pertenecen a la JFK Library constata que se utiliza la herramienta Twitter con finalidad autoreferencial y para fomentar la recuperación y consumo de los propios contenidos, ya que cada ficha facilita el acceso a otras páginas del mismo web de la JFK Library donde está clasificado el material archivado. El hecho de que en tan pocas ocasiones se enlace con páginas externas pone en evidencia que en este caso el uso de Twitter prioriza los contenidos autogenerados en lugar de facilitar el acceso a fuentes y medios externos. Por todo ello se puede concluir que, de las cinco funciones que esta red social lleva a cabo según la tipología de Xie y Stevenson (2014b), la cuenta @JFKsaid de la JFK Library sólo cumple la de fomentar la identidad social de la institución.

A partir del estudio de caso de la cuenta en Twitter de la JFK Library pueden extraerse algunas recomendaciones de buenas prácticas para el aprovechamiento de las redes sociales en bibliotecas. En particular, Twitter se revela como una herramienta útil para divulgar el conocimiento y potenciar el intercambio de información y documentación. El uso que hace la JFK Library de los recursos de fotografía, documentos de video, audio, texto y enlaces externos es generalizable a otras instituciones bibliotecarias, que pueden encontrar en esta red social un instrumento válido para interactuar con los usuarios y dar a conocer su patrimonio documental en un espacio interactivo, social y compartido. Sin embargo, creemos que la JFK Library podría haber sacado mucho más partido de Twitter y haber publicado muchos más tuits con enlaces a documentos digitalizados de su fondo documental. Recomendaríamos ampliar este uso divulgativo y educativo de la red de microblogging.

Si bien la distribución de los distintos tipos de archivos publicados a través de Twitter es más o menos proporcional a las existencias del fondo, recomendaríamos publicar muchos más videos, habida cuenta del poder de la imagen y su gran presencia en los medios sociales en pleno siglo XXI (es paradójico, como ya se mencionó, que se publique la transcripción digitalizada de la primera rueda de prensa de Kennedy televisada y no se publique el video, que existe en sus fondos).

Finalmente, recomendaríamos alejarse del uso autoreferencial, con el que se refuerza la función de fomento de la identidad social de la institución, y ampliar el acceso a fuentes y enlaces externos a la JFK Library, con lo que la función de conectividad social y de relación con otras fuentes aumentaría y 
se avanzaría en la construcción de comunidades en línea, uno de los principales valores de los medios sociales.

\section{REFERENCIAS}

Alonso Arévalo, Julio, José Antonio Cordón García, Raquel Gómez Díaz, Belén García-Delgado Giménez. 2014. "Uso y aplicación de herramientas 2.0 en los servicios de producción, organización y difusión de la información en la biblioteca universitaria". Investigación Bibliotecológica 28 (64): 51-74. https://www.researchgate.net/publication/269333396_Uso_y_aplicacin_de_herramieantas_2.0_ en_los_servicios_produccin_organizacin_y_difusin_de_la_informacin_en_la_ biblioteca_universitaria

Adams, Amelia, Tim McCorkindale. 2013. "Dialogue and transparency: A content analysis of how the 2012 presidential candidates use Twitter". Public Relations Review 39 (4): 357-359. http://doi:10.1016/j.pubrev.2013.07.016

Aharony, Noa. 2012. "Twitter use by three political leaders: an exploratory analysis". Online Information Review 36 (4):587-603. http://dx.doi. org/10.1108/14684521211254086

Aharony, Noa. 2010. "Twitter Use in Libraries: An Exploratory Analysis". Journal of Web Librarianship 4 (4): 333-350. http://dx.doi.rg/10.1080/19322909.2010.487766

Aharony, Noa. 2009. "Web 2.0 use by librarians". Library \& Information Science Research 31 (1): 29-37. http://dx.doi.org/10.1016/j.lisr.2008.06.004

Ammann, Sky L. 2010. "Why Do They Tweet? The Use of Twitter by U.S. Senate Candidates in 2010". Social Science Research Network. http://dx.doi.org/10.2139/ ssrn. 1725477

Boateng, Frank y Yan Quan Liu. 2013. "Web 2.0 applications' usage and trends in top US academic libraries". Library Hi Tech 32 (1): 120-138. http://dx.doi. org/10.1108/LHT-07-2013-0093

Casey, Michael E. y Laura C. Savstinuk. 2007. Library 2.0: a Guide to Participatory Library Service. Medford: Information Today Inc.

Chua, Alton Y. K. y Dion H. Goh. 2010. "A study of Web 2.0 applications in library websites". Library \& Information Science Research 32 (3): 203-211. http://dx.doi. org/10.1016/j.lisr.2008.06.004

Del Bosque, Darcy C., Sam A. Leif y S. Skarl. 2012. "Libraries Atwitter: Trends in Academic Library Tweeting". Reference Services Review 40 (2): 199-213. http:// dx.doi.org/10.1108/00907321211228246

Franch, Pere. 2013. "La retórica de Obama ante el terrorismo: estudio del uso de la red social Twitter tras los atentados de Boston de abril de 2013". adComunica. Revista Científica de Estrategias, Tendencias e Innovación en Comunicación 6: 83101. http://dx.doi.org/10.6035/2174-0992.2013.6.6

Giménez-Rayo, Mabel y Javier Guallar. 2014. "Centros de documentación en televisión y productos documentales". El profesional de la información 23 (1): 13-25. http://dx.doi.org/10.3145/epi.2014.ene.02 
Glassman, Matthew Eric, Jacob R. Straus y Colleen J. Shogan. 2010. "Social Networking and Constituent Communications: Member Use of Twitter During a Two-Month Period in the 111th Congress". Congressional Research Service. http://www.fas.org/sgp/crs/misc/R41066.pdf

Golbeck, Jennifer, Justin Grimes y Anthony Rogers. 2010. "Twitter use by the U.S. Congress". Journal of the American Society for Information Science and Technology 61 (8): 1612-1621. http://dx.doi.org/10.1002/asi.21344

Guallar, Javier. 2011. "La documentación en la prensa digital. Nuevas tendencias y perspectivas". III Congreso Internacional de Ciberperiodismo y Web 2.0, Bilbao: Universidad del País Vasco, 9-11 noviembre. http://eprints.rclis.org/16326/

Hoskins, Andrew. 2009. "The Digital Distribution of Memory". 1st Global Conference on Digital Memories, 1-15. http://www.inter-disciplinary.net/wp-content/ uploads/2009/03/hoskins-paper.pdf

JFK Library and Museum. 2009. "News Release. 50 Years Ago - Senator John F. Kennedy of Massachusetts Announces Candidacy for President of the United States". Press releases. News and Press. About us. https://www.jfklibrary.org/ About-Us/News-and-Press/Press-Releases/50-Years-Ago--Senator-John-F-Kennedy-of-Massachusetts-Announces-Candidacy-for-President-of-the-Unite.aspx

Kroski, Ellyssa. 2008. Web 2.0 for Librarians and Information Professionals. Nueva York: Neal-Schuman Publishers.

Mahmood, Khalid y John V. Richardson Jr. 2011. "Adoption of Web 2.0 in US academic libraries: a survey of ARL library websites”. Program 45 (4): 365-375. http:// dx.doi.org/10.1108/00330331111182085

Pinchevski, Amit. 2011. "Archive, Media, Trauma”. En On Media Memory: Collective Memory in a New Media Age, Mordechai Neiger, Oren Meyers y Eyal Zandberg, 253-264. London: Palgrave Macmillan.

Small, Tamara A. 2011. "What the hashtag? A content analysis of Canadian politics on Twitter". Information Communication E Society 14 (6): 872-895. http://dx.doi. org/10.1080/1369118x.2011.554572

Stephens, Michael. 2006. "Web 2.0 and Libraries: Best Practices for Social Software". Library Technology Reports 42 (4): 8-14.

Tripathi, Manorama y Sunil Kumar. 2010. "Use of Web 2.0 tools in academic libraries: A reconnaissance of the international landscape". The International Information \& Library Review 42 (3): 195-207. http://dx.doi.org/10.1016/j.iilr.2010.07.005

Tumasjan, Andranik, Timm O. Sprenger, Philipp G. Sandner e Isabell M. Welpe. 2010. "Predicting Elections with Twitter: What 140 Characters Reveal about Political Sentiment". Proceedings of the Fourth International Conference on Weblogs and Social Media. http://cs.wellesley.edu/ cs315/Papers/predicting elections with twitter.pdf

Xie, Iris y Jennifer A. Stevenson. 2014a. "Social media application in digital libraries”. Online information Review 38 (4): 502-523. http://dx.doi.org/10.1108/ OIR-11-2013-0261

Xie, Iris y Jennifer A. Stevenson. 2014b. "Functions of Twitter in Digital Libraries". 77th ASISET Annual Meeting, 1-4. https://www.asis.org/asist2014/proceedings/ submissions/posters/276poster.pdf 
Para citar este texto:

Franch, Pere y Elena Yeste. 2017. "Las redes sociales como herramienta para la divulgación de fondos documentales de bibliotecas. Estudio de caso de la cuenta en Twitter de la JFK Library". Investigación Bibliotecológica: Archivonomía, Bibliotecología e Información 72 (31): 21-37.

http://dx.doi.org/10.22201/iibi.0187358xp.2017.72.57822 